\title{
DINÁMICA FAMILIAR Y ENAMORAMIENTO DE PERSONAS CON DISCAPACIDAD
}

\author{
FREDDY PONCE \\ https://orcid.org/0000-0001-8762-7903 \\ Universidad Católica Boliviana San Pablo \\ La Paz, Bolivia \\ Correo electrónico: freddyponce29@yahoo.es
}

Recibido: 15 de marzo del 2021 / Aceptado: 31 de mayo del 2021

doi: https://doi.org/10.26439/persona2021.n024(1).5314

\begin{abstract}
Resumen. El objetivo de esta investigación es analizar teóricamente la dinámica familiar de personas con discapacidad en el proceso de enamoramiento para la conformación de una pareja. Estas personas enfrentan un entorno familiar que, por las creencias y prejuicios que sostienen, afectan negativamente su desarrollo social y afectivo. En este artículo se explora la experiencia amorosa de las personas con discapacidad para comprender la dinámica entre la persona con discapacidad y su familia. Como respuesta al desafío que estas personas encuentran a nivel individual y familiar, a partir del marco teórico sistémico social y psicoterapéutico, se propone formas de intervención, así como pautas para el acompañamiento en el proceso de enamoramiento.
\end{abstract}

Palabras clave: enamoramiento / personas con discapacidad / familia / afectividad

\section{FAMILY DYNAMICS AND FALLING IN LOVE IN PEOPLE WITH DISABILITIES}

\begin{abstract}
The purpose of this paper is to analyze the dynamics of families that include a member with disabilities who is in the process of falling in love and having a relationship. This member will experience a negative impact from her/his family, due to certain beliefs and prejudices, that will hinder in her/his social and emotional development on his/her development. In this article, we explore the love experience in individuals with disabilities to understand the dynamics between family and the member with disabilities. As a result of the challenges these people encounter at the individual and family level; I propose guidelines, based on the theoretic and systemic social and psychotherapeutic frame, for the intervention, as well as rules for the follow up in the process of engaging in love.
\end{abstract}

Keywords: falling in love / feelings / family / people with disabilities 


\section{DISCAPACIDAD Y FAMILIA}

La discapacidad es constantemente debatida y, sin embargo, no encontramos una definición consolidada que permita su estudio desde diferentes modelos. Mientras que el modelo individual conceptualiza la discapacidad como un problema médico que genera un impedimento, el modelo social reconoce la discapacidad como una barrera que coloca a la persona en una minoría en la que su vivencia se ve influenciada por el entorno y no solamente por su condición (Almario, 2016; Pérez y Chhabra, 2019). En la discapacidad se reconoce a toda persona con una limitación física, mental, intelectual o sensorial que puede ser congénita o adquirida en el tiempo que determina la vivencia del individuo dentro de la sociedad (Abad-Salgado, 2016; Palacios, 2017).

Además de las dificultades que el individuo afronta producto de su condición, también se encuentran varios problemas sociales y familiares. A nivel social se encuentra la falta de formación de profesionales para la inclusión de estas personas en espacios o actividades, la demanda de recursos para la intervención o gestión de esta problemática y la participación de esta población dentro la sociedad en un ámbito económico, político o educativo sin exclusión. Entonces, no se evidencia el cumplimiento o mejora de sus condiciones básicas y calidad de vida o brindar igualdad de oportunidades (Martín y Sánchez, 2016). La familia de una persona con discapacidad es la institución social encargada de su cuidado y protección, y por ello asumen un rol protagónico, puesto que servirán de apoyo para solventar sus necesidades y demandas para su convivencia. Sin embargo, la persona con discapacidad no es, necesariamente, el hijo o la hija, sino una madre, padre o algún miembro de la familia ampliada que se incorpora al núcleo familiar.

El diagnóstico de discapacidad impacta en el sistema familiar, rompe su armonía y afecta las relaciones conyugales, fraternas y filiales (Abad-Salgado, 2016; Villavicencio, Romero, Criollo y Peñaloza, 2018). La reacción al diagnóstico puede traducirse en tensiones, estrés familiar, hipersensibilidad o labilidad emocional, incertidumbre, culpa, desilusión y tristeza que se convierte, finalmente, en la vivencia de un duelo familiar (Villavicencio et al., 2018). De esta manera, la dinámica familiar retrata un duelo o pérdida de las expectativas que se tenía con un hijo o miembro familiar. Este duelo se caracterizará por producir un desgaste emocional, aislamiento frente a su entorno y déficits para tomar acciones ante la discapacidad. Por consiguiente, se desarrollarán patrones de sobreprotección o asistencialistas hacia la persona con discapacidad influenciados también por un entorno que elabora juicios de valor (Almario, 2016).

El duelo en la familia es una pérdida ambigua, puesto que no hay una muerte física del individuo con discapacidad, sino incertidumbre acerca de su futuro. Por esta razón, el afrontamiento de este proceso se bloquea o congela, y el cierre se torna difícil o en ocasiones imposible (Boss, 2001; Quiroz-Chagoya, 2017). Se entiende este duelo como un proceso para sobrellevar la pérdida. Kubler-Ross (1969) plantea etapas del duelo, pero 
luego se ha establecido como un proceso sin un orden definido en el que es necesario cumplir tareas para superarlo (Worden, 2004).

Se destacan tres procesos que vive la familia cuando se establece la discapacidad en uno de sus miembros, estos son: la desintegración que se afronta con cólera, culpa, ansiedad y se reacciona con shock; negación-aceptación de la realidad y reintegración donde se reorganizan roles y funciones. Cuando la familia queda atrapada en estas etapas se desconoce al individuo como persona y se coloca como objeto de cuidado desconociendo sus demandas. En la familia, esta situación muestra una inadecuada elaboración del duelo (Villavicencio et al., 2018).

El duelo no resuelto en la familia se ve influenciado por creencias o normas sociales generadas y reproducidas entre el Estado, la familia y el mismo individuo. Se estigmatiza la forma de vida e interacción de la persona con discapacidad con su entorno. Así, el individuo con discapacidad será vulnerable a la discriminación, abuso físico o psicológico, incomprensión, falta de tolerancia y restricciones o limitaciones en la toma de decisiones o en la satisfacción de ciertas necesidades personales dentro su núcleo familiar (Polanco y Martín, 2017).

Las creencias o normas sociales configurarán determinantes de salud con desventajas para el individuo; se da cuenta de barreras físicas, estructurales y culturales que limitan la vida de la persona con discapacidad en vez de generar igualdad (Tamayo, Besoaín y Rebolledo, 2018). Este determinante social se transmite a la familia por profesionales e instituciones como una representación negativa de la calidad de vida de las personas con discapacidad, influido en algunos casos por modelos religiosos y médicos que presentan una visión parcial del fenómeno (Martín y Sánchez, 2016; Losada y Muñiz, 2019).

La dinámica familiar, al quedar atrapada en una etapa del duelo, no permite una buena adaptación a la situación y a su entorno (Villavicencio et al., 2018). En consecuencia, se produce un proceso de desubjetivización en la familia en el que se aniquila o se niega la situación existente. En otros casos, la persona con discapacidad permanece como un objeto de cuidado o un niño eterno en la familia (Abad-Salgado, 2016; Martín y Micheletti, 2019). Todo esto lleva a un patrón de sobreprotección o asistencialismo (Almario, 2016), y en el caso de algunas instituciones, al maltrato basado en relaciones de poder y prejuicios (Linares, 2002).

La dinámica familiar influye en la toma de decisiones con respecto a su forma de vida, en las relaciones que establece con su entorno e inclusive en sus actividades cotidianas. Los patrones de cuidado o crianza a la persona con discapacidad están determinados por mitos familiares, estrategias de afrontamiento y la participación de los miembros del núcleo familiar o de la familia ampliada. Entre las estrategias de afrontamiento internas de la familia se encuentra la evaluación pasiva, que es el reconocimiento 
de la nueva situación, la restructuración y la movilización familiar (Quintero, Amaris y Pacheco, 2020).

Esta dinámica familiar genera en la persona con discapacidad una posición de objeto, que se caracteriza por ser circular y se retroalimenta a sí misma, puesto que la desubjetiviza. Tanto el temor como la discriminación hacia la discapacidad determinan los patrones de cuidado o la sobreprotección, desubjetiviza al individuo influenciado por una familia que tiene miedo y discrimina a la discapacidad. Estos procesos solo confirman y retroalimentan la desubjetivación y permanencia, volviéndose un ciclo en el que la familia tiene un rol eterno de cuidado y sobreprotección. Esta dinámica se representa en la figura 1.

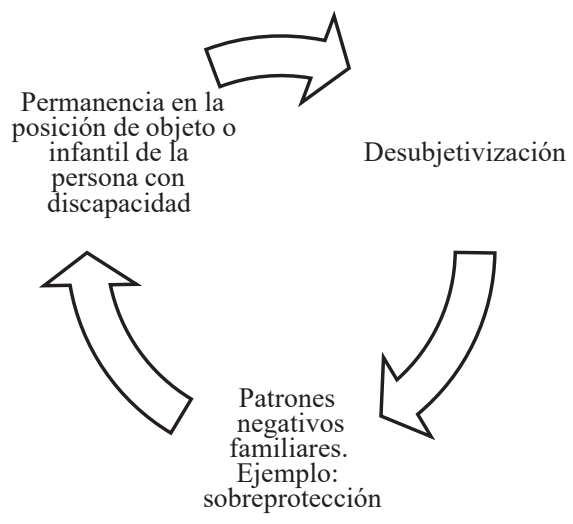

Figura 1. Dinámica familiar en una persona con discapacidad

Elaboración propia

\section{AMOR, AFECTIVIDAD Y ENAMORAMIENTO EN LA DISCAPACIDAD}

El enamoramiento de una persona con discapacidad no es ajeno a la familia porque este proceso individual puede ser sinónimo de un abandono del nido y una crisis normativa en el sistema. Además de las barreras que enfrentan la persona con discapacidad y su familia, se suma la vivencia de crisis normativas (Almario, 2016). La familia ocupa una posición importante en el enamoramiento de la persona con discapacidad dado que influye directamente en las condiciones de vida de la persona, sus redes de apoyo (amigos, compañeros y profesionales) y espacios de desenvolvimiento (Martín y Sánchez, 2016).

El enamoramiento es un proceso que se inicia con la elección de pareja con base en la atracción y el deseo. Sin embargo, el hecho de haber elegido una pareja no significa que sea correspondido y más bien puede llevar a una pérdida ambigua de la persona 
idealizada. Este proceso se afianza con la conquista o cortejo amoroso que depende de los recursos del individuo para llegar al amado o amada (Ponce y Pinto, 2018). Si es correspondido, este enamoramiento se caracterizará por ser adictivo, obsesivo, apasionado y tierno, con la intervención biológica de la adrenalina, dopamina y acetilcolina por el estrés vivido. Entonces, la elección de pareja, el cortejo amoroso y el enamoramiento es un proceso que depende de condiciones sociales y biológicas (Pinto, 2012).

En el enamoramiento y el proceso de conformación de pareja de las personas con discapacidad, se han encontrado relaciones románticas llenas de placer y fascinación. Se trata de una etapa cristalizante, en la que solo le interesará la compañía y la pasión por el otro. Al mismo tiempo, ayudará al crecimiento o superación personal con el desarrollo de un amor maduro (Mattila, Uusiautti y Maatta, 2017). Sin embargo, se ha observado también mayor ansiedad. En muchos casos, la tensión, inseguridad y vulnerabilidad aparecen porque no se cuenta con redes de apoyo como amigos o familiares para la orientación o acompañamiento en los procesos de conquista, sexualidad, concepción de amor y la búsqueda de pareja. De este modo, la persona con discapacidad tendrá que desarrollar estrategias de afrontamiento y lidiar con sus emociones para superar este proceso de manera individual (Loeser, Pini y Crowley, 2017; Puyaltó, Pallisera, Fullana y Díaz, 2019).

Se ha encontrado en las personas con discapacidad las reacciones físicas evidentes como el latido fuerte del corazón, cambios de humor o señales de afecto agresivas o cordiales hacia la persona de interés (Mattila et al., 2017) que son malinterpretadas por la familia o las redes de apoyo (compañeros, amigos o profesionales). De esta forma, no se genera la contención emocional o espacios para la comunicación y expresión de emociones que favorezcan el bienestar emocional (Huaiquián, Arriagada, Betanzo, Inostroza y Llanquitruf, 2018).

En el enamoramiento, la afectividad es sumamente importante porque constituye uno de los conductores en el comportamiento para la expresión de emociones, sentimientos, motivaciones y deseos hacia el otro. Las manifestaciones de afectividad en las personas con discapacidad están determinadas, al igual que todo el proceso de enamoramiento, por la cultura, la educación y la religión. Las formas que adquiere la afectividad en el enamoramiento de personas con discapacidad se dan mediante el cariño, dar importancia a la otra persona sobre uno, obsequios, expresiones corporales, compañía, idealización y posicionamiento del otro frente a los demás (Huaiquián et al., 2018).

Las expresiones de afecto se entienden como momentos de intimidad con las otras personas que son limitados por las instituciones o las familias. Se suele adoptar una actitud hipervigilante y separar a las mujeres y varones en grupos (Puyaltó et al., 2019). Esto se traduce en un desconocimiento de los derechos de la persona con discapacidad porque la familia o los profesionales asumen la responsabilidad de con quién o cómo se relacionan, así como respecto a su sexualidad (Ignagni, Fudge, Liddiard y Runswick, 2016). 
Independientemente del enamoramiento y la afectividad, el amor en parejas de personas con discapacidad solo se dará en la consolidación de este sistema de a dos de manera formal que comienza usualmente con la amistad (Puyaltó et al., 2019). Se ama solo en un sistema dual en el que se legitima al otro en una relación recíproca (Pinto, 2012). Desde la teoría triangular del amor de Sternberg (2000), los componentes del amor son la pasión, la intimidad y el compromiso. El componente de pasión tiene dos formas: una erótica y otra romántica (Yela, 2002). Estos componentes matizarán la forma, dinámica y experiencia amorosa dependiendo de cuál es el componente que se destaca en cada persona y que conformará su concepto de amor. Morentin, Verdugo, Arias y Rodríguez (2006) establecen que en las personas con discapacidad los componentes del amor aparecen en el siguiente orden: primero la intimidad, seguida de la pasión y, finalmente, el compromiso.

La intimidad supone un espacio para el desarrollo de un vínculo o conexión de confianza en el que encuentra un apoyo emocional y comunicación con otra persona fuera de su círculo habitual (Sternberg, 2000; Morentin et al., 2006). La intimidad resulta importante para el desarrollo de habilidades sociales, empatía y apertura (Mattila et al., 2017). La pasión hace referencia a la manifestación o expresión de afectos sobre la satisfacción de un deseo sexual (Sternberg, 2000) e incluye las expresiones físicas de afecto como los cariños y besos o la compañía. Estas serán claves para comprender la naturaleza de la relación y la principal preferencia de las personas con discapacidad (Bates, Terry y Popple, 2016). El compromiso es el componente del amor que implica la decisión de amar a otra persona y el mantenimiento de la relación relacionado a un contrato (Sternberg, 2000). El desafío en personas con discapacidad, más allá de encontrar a la persona correcta, está en tener momentos de intimidad, lograr independencia de las familias de origen, la manutención de la relación de forma económica, planificación familiar y de pareja (Puyaltó et al., 2019).

Según la teoría de estilos de amor desarrollada por Hendrick y Hendrick (1989), se identifican seis estilos de amor: eros (amor apasionado), storge (amor de amigos), ludus (amor de entretenimiento), manía (amor posesivo), pragma (amor práctico) y ágape (amor desinteresado). En efecto, el amor en la discapacidad se caracterizará por ser del tipo de amigos, entretenimiento o apasionado por la prevalencia de los componentes de intimidad y pasión. Las relaciones amorosas, entonces, en personas con discapacidad se destacarán por ser más afectivas que sexuales, sin diferencias entre varones y mujeres. Sin embargo, el componente "compromiso" se sobrepone sobre los otros dos cuando la persona con discapacidad es adulta (Morentin et al., 2006; Bates et al., 2016).

En síntesis, el amor, la afectividad y la vivencia del enamoramiento son de vital importancia para el desarrollo emocional, social e individual de las personas con discapacidad. Independientemente de la edad, ellos o ellas aprenden acerca de la compañía fuera de la familia, satisfacen necesidades emocionales, establecen relaciones de 
confianza y mejoran su estatus social; en conjunto, la experiencia les proporciona una sensación de normalidad. La experiencia de enamoramiento o de pareja en las personas con discapacidad permite romper barreras internas y externas al confrontar la exclusión social, abuso psicológico o físico de su familia, sentimiento de soledad o aislamiento (Bates et al., 2016).

El enamoramiento y la convivencia de pareja en personas con discapacidad suponen un desafío a nivel individual, familiar y social. Supone que la familia e instituciones se enfrenten a prejuicios o creencias acerca de la discapacidad y se consoliden como factores de apoyo y de protección a la persona con discapacidad; el enamoramiento o el establecimiento de relaciones de pareja no se puede evitar y se debe enfrentar el cambio (Mattila et al., 2017; Losada y Muñiz, 2019). En algunos casos, ocurre la ruptura de las relaciones de pareja o aparecen situaciones de amor no correspondido en las personas con discapacidad. Estas generan una pérdida ambigua donde se trata de sobrellevar la ausencia o rechazo de la otra persona que se define como un duelo amoroso (Ponce y Pinto, 2018). El rol de la familia o la institución cobra especial importancia para trascender esta experiencia de ruptura o rechazo y facilitar que esta sea una fase de aprendizaje acerca de las relaciones de pareja para la persona con discapacidad (Bates et al., 2016).

\section{¿QUÉ HACER EN EL ENAMORAMIENTO DE UNA PERSONA CON DISCAPACIDAD?}

Con el objeto de dar curso y apoyar el enamoramiento en una persona con discapacidad se establece la necesidad de intervenir o trabajar con la familia y la persona para que no se restrinja este proceso y se generen conflictos individuales posteriores. Ya que las oportunidades para la conformación de relaciones de pareja en personas con discapacidad son pocas, tanto la familia como las personas con discapacidad deben reconocer el potencial de desarrollo de este proceso (Bates, Terry y Popple, 2017).

\section{Intervención familiar}

La intervención familiar se basa en trabajar el duelo o pérdida ambigua que tiene una influencia negativa en el proceso de enamoramiento de personas con discapacidad porque se restringe la experiencia y en algunos casos se la invisibiliza. Entonces, esta dinámica debe cambiar para velar por su equilibrio social, físico, psíquico y emocional (Abad-Salgado, 2016). Resulta importante que se culmine el proceso de duelo con una reorganización o reintegración del sistema familiar de modo que sea flexible, que se dé la rotación de responsabilidades para el cuidado o la atención de la persona con discapacidad, que se agradezca o reconozca al cuidador y se generen espacios para la comunicación (Villavicencio et al., 2018).

Para favorecer un proceso de enamoramiento es importante dar por finalizado el duelo en la familia mediante la realización de tareas. Estas tareas deben estar enfocadas 
a una pérdida ambigua y romper una dinámica familiar que ha hecho del sistema familiar un espacio homeostático y dañino para la persona con discapacidad. Esta dinámica se encuentra al interior del sistema, pero también se observa en cada uno de los miembros un proceso de duelo individual, como se advierte en la figura 2.

Permanencia en la posición de objeto o infantil de la persona con discapacidad

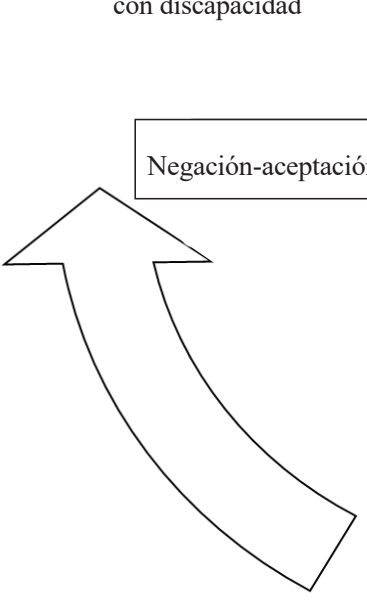

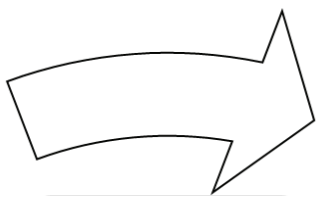

Desintegración
Desubjetivización

Figura 2. Duelo familiar: eje problemático y dinámica familiar

\section{Elaboración propia}

Así, además del duelo familiar grupal, se identifican procesos de pseudonegación y aceptación para cada uno de sus miembros. Por lo que en el sistema familiar en su conjunto aparece la desubjetivización, patrones negativos de funcionamiento y la permanencia de posición de objeto o infantilización de la persona con discapacidad. La aceptación o negación de la discapacidad surge como el principal punto de conflicto y puede dar lugar a una dinámica familiar perjudicial que involucra a todos los miembros de la familia.

Cabe recalcar que no solo se afecta a la persona con discapacidad, en algunos casos afecta también al cuidador primario o la relación de pareja de la familia. Por lo que se debe velar por las necesidades de toda la familia, de los individuos parte de esta y de la misma persona con discapacidad (Almario, 2016). Con este fin, las tareas para trabajar en la familia son, como plantea Worden (2004), la diferenciación de necesidades 
familiares e individuales, la identificación o experimentación de sentimientos, examinar defensas o estilos de afrontamiento y la recolocación de la persona con discapacidad.

Estas tareas se han visto reflejadas en la instauración de procesos terapéuticos o encuentros entre la familia y la persona con discapacidad en la que se trabajen las necesidades y demandas afectivas (Bates et al., 2017). Se busca movilizar a la familia hacia una nueva dinámica en la que se subsane y se llegue a acuerdos dando paso a procesos de perdón y reencuentro. Además, resulta beneficioso para la familia incorporar a los padres o cuidadores primarios a los grupos de apoyo para la orientación, soporte o entrenamiento o intercambio de experiencias brindados por fundaciones $u$ organizaciones de manera online o presencial (Bates, 2019).

Los resultados de la intervención en la familia se evidencian con la construcción de vínculos afectivos sin violencia o carga de creencias negativas o prejuicios, así como a través de procesos de socialización con el entorno. También con la facilidad para la reacomodación ante las crisis y un rol de cuidador primario dinámico que no se recarga solo en una persona o miembro de la familia (Polanco y Martín, 2017). En el enamoramiento de la persona con discapacidad, la familia ocupa una posición de guía, orientación o soporte para la planificación familiar, sexualidad, dinámica del amor o las relaciones de parejas sanas. A pesar de que las familias sienten miedo o ansiedad ante la situación, podrían manejarla en beneficio de la persona con discapacidad.

Las formas de intervención familiar pueden ser desde el apoyo psicológico mediante la psicoterapia familiar o grupal, además de una intervención psicosocial grupal. La psicoterapia familiar es un procedimiento específico para trabajar con la familia como conjunto sobre la base de sus características y problemas, necesidades y demandas. La psicoterapia grupal constituye un espacio de encuentro para compartir experiencias con la presencia de los miembros principales de familias con personas con discapacidad. Usualmente, esta busca el crecimiento de la familia y el participante mediante el intercambio dinámico que permita sobrellevar un proceso en conjunto con otras familias.

La intervención psicosocial grupal, por su parte, es un proceso no específicamente terapéutico guiado por profesionales de distintas áreas que busca brindar apoyo social a la familia y las personas con discapacidad. Usualmente, esta forma de intervención utilizará una red social formal o informal mediante instituciones o servicios. Estas son sesiones en red o reuniones de socialización en las que se busca brindar información y resolver problemas de la familia que escapan de su control (Navarro y Rodríguez, 2017).

Dicha intervención puede incluir programas para la educación sexual o familiar dirigidos a la persona con discapacidad o a los padres o cuidadores (Loeser et al., 2017; Huaiquián et al., 2018). Se cuenta, además, con protocolos de intervención psicosocial más a largo a plazo como son las escuelas para padres, programas dirigidos a apoyar dimensiones de crianza, comunicación, cuidado y protección a los hijos, crisis normativas o el 
ciclo vital familiar; en algunos casos, se basan en algunos casos en problemáticas específicas para el manejo dentro el hogar o la familia mediante la orientación y consejería.

\section{Intervención individual a la persona con discapacidad}

La intervención individual a la persona con discapacidad busca brindar soporte, apoyo psicológico y concientización durante este proceso o la vivencia con su condición. Por un lado, se orienta al desarrollo de sus fortalezas cognitivas o físicas con especialistas según su discapacidad. Por otro lado, se realiza un trabajo psicoterapéutico donde se atienden las demandas de la persona con discapacidad y las crisis o problemas propios del ciclo vital individual. También se requiere apoyo psicosocial para la concientización con respecto a la planificación familiar, sexualidad, relaciones de amistad o pareja, educación, orientación vocacional y hábitos.

La persona con discapacidad, en efecto, debe desarrollar autonomía e independencia en su forma de vida pese a su condición; estos constituyen los principales signos de estabilidad y buen desarrollo individual. La autonomía e independencia se reflejan en la toma de decisiones y el autocuidado, tratando de evitar la dependencia de su familia o entorno. Pese a esto, también se busca desarrollar estrategias de afrontamiento en su salud mental al ser vulnerables a otras enfermedades o condiciones. En otros casos, el fomento de la autonomía e independencia permite optimizar los recursos individuales de la persona con discapacidad (Abad-Salgado, 2016).

Usualmente, las demandas de la persona con discapacidad en la familia o instituciones no son tomadas en cuenta y están orientadas solo al cuidado o satisfacción de sus necesidades básicas. Las principales demandas son las de cuidado, supervisión y de afecto. Las demandas de cuidado y supervisión se cumplen a través de instituciones, redes sociales o la misma familia (Villavicencio et al., 2018). Sin embargo, la no satisfacción de las demandas afectivas produce desesperanza y tristeza. Al no hallar consuelo, estas emociones pueden ser exacerbadas o la persona puede buscar alternativas para satisfacer o bloquear sus demandas, aprendiendo a no lidiar con su afectividad.

El manejo de la afectividad en la persona con discapacidad es un elemento clave en la intervención individual y requiere la realización de un acompañamiento en este proceso tanto a nivel psicoterapéutico como social. El acompañamiento puede realizarse preferentemente por personas cercanas o significativas para la persona con discapacidad o por un profesional que brinde apoyo psicológico. Pueden surgir problemas con el rechazo o la ruptura amorosa cuando la persona lidia con un duelo y se deben trabajar las emociones, decisiones y causas. Por otro lado, si las relaciones funcionan, es necesario trabajar con la persona la planificación familiar, la educación sexual, fortalezas o debilidades de la relación y hacer seguimiento a la toma de decisiones. 
Aunque la intervención familiar o individual es un desafío cuando se trata la discapacidad, esta es necesaria porque se encuentran dinámicas que generan problemas tanto individuales o grupales que son comunes en este tipo de población y muestran la problemática que viven. Todo esto repercute en el establecimiento de relaciones de pareja en personas con discapacidad, pues organizan las condiciones, medios y espacios para su desenvolvimiento. Pero esta lectura da a conocer que más allá de realizar un acompañamiento al proceso de enamoramiento, con la intervención se debe trabajar el eje del problema que es el duelo en la familia.

En este sentido, se recomienda explorar más a profundidad esta dinámica en la familia, en el individuo con discapacidad y en el cuidador primario. Por otro lado, se necesita sistematizar y dar ejemplos de los modelos de intervención planteados tanto a nivel individual y familiar. Otro aspecto a tomar en cuenta es que solo se explora el enamoramiento en la discapacidad cuando puede haber otros procesos individuales o familiares que son afectados por la retroalimentación negativa familiar. De este modo, es necesario prestar atención, a nivel de investigación cualitativa o cuantitativa, a aspectos como la orientación vocacional o proyecto de vida y el desarrollo de amistades o relaciones sociales. Estas recomendaciones son necesarias para explorar la temática y los problemas circundantes a esta, como también sensibilizar acerca de la discapacidad.

\section{FINANCIAMIENTO}

El presente trabajo fue autofinanciado por el autor.

\section{CONFLICTO DE INTERESES}

El autor expresa que no existe un conflicto de interés en la redacción de este manuscrito.

\section{REFERENCIAS}

Abad-Salgado, A. M. (2016). Familia y discapacidad: consideraciones apreciativas desde la inclusión. Revista Latinoamericana de Estudios de Familia, 8, 58-77. http:// revlatinofamilia.ucaldas.edu.co/downloads/Rlef8_5.pdf

Almario, E. O. (2016). La investigación en familias de personas con discapacidad. Paideia Surcolombiana, 21, 12-26. https://doi.org/10.25054/01240307.1443

Bates, C. (2019). Supported Loving- Developing a National Network to Support Positive Intimate Relationships for People with Learning Disabilities. Tizard Learning Disability Review, 24(1), 13-19. https://doi.org/10.1108/TLDR-06-2018-0017 
Bates, C., Terry, L., y Popple, K. (2016). The Importance of Romantic Love to People with Learning Disabilities. British Journal of Learning Disabilities, 45(1), 64-72. https:// doi.org/10.1111/bld.12177

Bates, C., Terry, L., y Popple, K. (2017). Supporting People with Learning Disabilities to Make and Maintain Intimate Relationships. Tizard Learning Disability Review, 22(1), 16-23. https://doi.org/10.1108/TLDR-03-2016-0009

Boss, P. (2001). La pérdida ambigua. Gedisa.

Hendrick, C., y Hendrick, S. (1989). Research of Love: Does it Measure Up? Journal of Personality and Social Psychology, 56(5), 784-794. https://doi.org/10.1037/00223514.56.5.784

Huaiquián, C. B., Arriagada, C. V., Betanzo, A. B., Inostroza, S. H., y Llanquitruf, P. K. (2018). Manifestaciones afectivas en jóvenes con discapacidad intelectual. Interdisciplinaria, 35(1), 69-86. http://www.redalyc.org/articulo. oa?id=18058784004

Ignagni, E., Fudge, A., Liddiard, K., y Runswick, K. (2016). "Some People are not Allowed to Love": Intimate Citizenship in the Lives of People Labelled with Intellectual Disabilities. Disability \& Society, 31(1), 131-135. https://doi.org/10.1080/0968759 9.2015 .1136148

Kubler-Ross, E. (1969). On Death and Dying. Macmillan. http://www.worldcat.org/title/ on-deaath-and-dying/oclc/4238

Linares, J. L. (2002). Del abuso y otros desmanes. El maltrato familiar, entre la terapia y el control. Ediciones Paidós.

Loeser, C., Pini, B., y Crowley, V. (2017). Disability and Sexuality: Desires and Pleasures. Sexualities, 21(3), 255-270. https://doi.org/10.1177/1363460716688682

Losada, A. V., y Muñiz, A. M. (2019). Sexualidad en sujetos con discapacidad intelectual. Mitos y prejuicios como factores de riesgo y vulnerabilidad al abuso sexual. Revista Digital Prospectivas en Psicologia, 3(2), 4-15. https://www.kennedy.edu.ar/ wp-content/uploads/2019/10/2019_07_Revista-Prospectiva_carta.pdf\#page=4

Martín, A., y Micheletti, A. (2019). Niñez eterna. La infantilización en la discapacidad intelectual. F@ ro: Revista Teórica del Departamento de Ciencias de la Comunicación, 1(29), 5-23. https://dialnet.unirioja.es/servlet/articulo?codigo=7070507

Martín, M. V. C., y Sánchez, M. C. G. (2016). Análisis cualitativo de tópicos vinculados a la calidad de vida en personas con discapacidad. Ciência y Saúde Coletiva, 21, 23652374. https://doi.org/10.1590/1413-81232015218.04182016 
Mattila, J., Uusiautti, S., y Maatta, K. (2017). How do People with Intellectual Disability Describe the Experience of Falling in Love? International Journal of Emotional Education, 9(1), 71-84. https://www.um.edu.mt/library/oar//handle/123456789 $/ 18279$

Morentin, R., Verdugo, M. A., Arias, B., y Rodríguez, J. M. (2006). Amor y enamoramiento en personas con discapacidad intelectual: un campo por explorar. Siglo Cero: Revista Española sobre Discapacidad Intelectual, 37(217), 59-80. https://dialnet. unirioja.es/servlet/articulo?codigo $=1998060$

Navarro, J. G., y Rodríguez, J. A. (2017). Intervención en la red social de la familia con personas con discapacidad. Mosaico: Revista de la Federación Española de Asociaciones de Terapia Familiar = Journal of the Spanish Federation of Family Therapy Associations, 66, 31-45.

Palacios, A. (2017). El modelo social de discapacidad y su concepción como cuestión de derechos humanos. Revista Colombiana de Ciencias Sociales, 8(1), 14-18. https:// doi.org/10.21501/22161201.2190

Pérez, M. E., y Chhabra, G. (2019). Modelos teóricos de discapacidad: un seguimiento del desarrollo histórico del concepto de discapacidad en las últimas cinco décadas. Revista Española de Discapacidad (REDIS), 7(1), 7-27. https://dialnet.unirioja.es/ servlet/articulo?codigo $=6955448$

Pinto, B. (2012). Psicología del amor. Departamento de Psicología de la Universidad Católica Boliviana "San Pablo".

Polanco, M. V., y Martín, J. L. (2017). Conocimientos, actitudes y prácticas de familias de adolescentes con discapacidad cognitiva en sexualidad y afectividad. Diversitas: Perspectivas en Psicología, 13(2), 187-199. https://doi.org/10.15332/s17949998.2017.0002.04

Ponce, F. A., y Pinto, B. (2018). Cortejo amoroso en un grupo de jóvenes varones de la ciudad de La Paz. Ajayu Órgano de Difusión Científica del Departamento de Psicología UCBSP, 16(1), 123-149. http://www.scielo.org.bo/scielo.php?pid=S2077$21612018000100005 y$ script=sci_arttextytlng=pt

Puyaltó, C., Pallisera, M., Fullana, J., y Díaz, G. (2019). Challenges of Having a Loving Partner: The Views of Adults with Intellectual Disabilities. International Journal of Developmental Disabilities. https://doi.org/10.1080/20473869.2019.1701786

Quintero, F. J., Amaris, M. D. C., y Pacheco, R. A. (2020). Afrontamiento y funcionamiento en familias en situación de discapacidad. Revista Espacios, 41(17), 21-30. http:// ww.revistaespacios.com/a20v41n17/a20v41n17p21.pdf 
Quiroz-Chagoya, M. Á. (2017). Del duelo a la pérdida ambigua: un análisis sobre el duelo tras la presencia de discapacidad en hijos y/o hijas. Revista Electrónica de Educación Especial y Familia, 1(8), 74-85.

Sternberg, R. J. (2000). La experiencia del amor. La evolución de la relación amorosa a lo largo del tiempo. Paidós.

Tamayo, M., Besoaín, Á., y Rebolledo, J. (2018). Determinantes sociales de la salud y discapacidad: actualizando el modelo de determinación. Gaceta Sanitaria, 32(1), 96-100. https://doi.org/10.1016/j.gaceta.2016.12.004

Villavicencio, C. E. A., Romero, M. M., Criollo, M. A., y Peñaloza, W. P. (2018). Discapacidad y familia: desgaste emocional. Academo: Revista de Investigación en Ciencias Sociales y Humanidades, 5(1), 89-98. https://doi.org/10.30545/academo.2018. ene-jun.10

Worden, W. J. (2004). El tratamiento del duelo: asesoramiento psicológico y terapia (2. ${ }^{a}$ ed). Ediciones Paidós.

Yela, C. (2002). El amor desde la psicología social. Ni tan libres, ni tan racionales. Pirámide. 